\title{
The comparison of the surgical outcome of 27-gauge pars plana vitrectomy for primary rhegmatogenous retinal detachment between air and SF6 gas tamponade
}

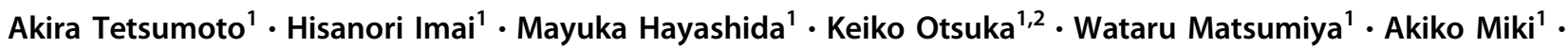 \\ Makoto Nakamura ${ }^{1}$
}

Received: 12 June 2019 / Revised: 8 August 2019 / Accepted: 8 September 2019 / Published online: 20 December 2019

(c) The Author(s), under exclusive licence to The Royal College of Ophthalmologists 2019

\section{Learning objectives}

Upon completion of this activity, participants will be able to:

1. Compare anatomical success rate between air and 20\% SF6 tamponade in 27GPPV for RRD, according to a retrospective, observational, and consecutive study.

2. Compare visual outcomes and changes in intraocular pressure during 12-month follow-up between air and $20 \%$ SF6 tamponade in 27GPPV for RRD, according to a retrospective, observational, and consecutive study.

3. Compare type and frequency of complications during 12-month follow-up between air and 20\% SF6 tamponade in $27 \mathrm{GPPV}$ for RRD, according to a retrospective, observational, consecutive study, and describe other clinical implications regarding surgical outcomes.

\section{Accreditation Statements}

In support of improving patient care, this activity has been planned and implemented by Medscape, LLC and Springer Nature. Medscape, LLC is jointly accredited by the Accreditation Council for Continuing Medical Education (ACCME), the Accreditation Council for Pharmacy Education (ACPE), and the American Nurses Credentialing Center (ANCC), to provide continuing education for the healthcare team.

Medscape, LLC designates this Journal-based CME activity for a maximum of 1.0 AMA PRA Category 1 Credit(s) ${ }^{\mathrm{TM}}$. Physicians should claim only the credit commensurate with the extent of their participation in the activity.

\section{Credit Hours}

1.0

Release date: 20 December 2019

Expiration date: 20 December 2020

Post-test link: https://medscape.org/eye/posttest 921555

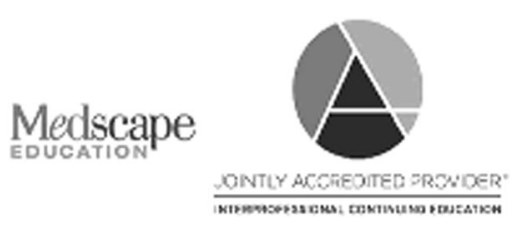

\section{Authors/Editors disclosure information}

A.T. has disclosed no relevant financial relationships. H.I. has disclosed the following relevant financial relationships: received grants for clinical research from: Alcon Japan. M.H. has disclosed no relevant financial relationships. K.O. has disclosed no relevant financial relationships. W. M. has disclosed no relevant financial relationships. A.M. has disclosed no

Hisanori Imai

hisimai@med.kobe-u.ac.jp

1 Department of Surgery-Related, Division of Ophthalmology, Kobe University Graduate School of Medicine, 7-5-2 Kusunoki-cho, Chuo-ku, Kobe 650-0017, Japan
2 Kobe Kaisei Hospital, 3-11-15 Shinohara Kitamachi, Nada-ku, Kobe 657-0068, Japan 
relevant financial relationships. M.N. has disclosed the following relevant financial relationships: received grants for clinical research from: Alcon Japan; Otsuka Pharmaceuticals. Sobha Sivaprasad (Editor) has disclosed the following relevant financial relationships: Served as an advisor or consultant for: Allergan, Inc.; Bayer AG; Boehringer Ingelheim Pharmaceuticals, Inc.; Heidelberg Pharma GmbH; Optos; Roche. Served as a speaker or a member of a speakers bureau for: Allergan, Inc.; Bayer AG; Novartis Pharmaceuticals Corporation; Optos. Received grants for clinical research from: Allergan, Inc.; Bayer AG; Boehringer Ingelheim Pharmaceuticals, Inc.; Novartis Pharmaceuticals Corporation; Optos.

\section{Journal CME author disclosure information}

Laurie Barclay has disclosed no relevant financial relationships.

\section{Abstract}

Objectives To compare the surgical results between air and 20\% sulfur hexafluoride (SF6) tamponade in 27-gauge pars plana vitrectomy (27GPPV) for rhegmatogenous retinal detachment (RRD).

Methods A retrospective, observational, and consecutive study. All patients underwent 27GPPV for RRD were divided into two groups. Group A comprised patients who underwent 20\% SF6 gas tamponade. Group B comprised patients who underwent air tamponade. The anatomical success rate, visual outcome, and the type and frequency of complications were investigated and compared between the groups. All patients were followed-up for 12 months after surgery.

Results Seventy eyes were enrolled (Group A: 35 eyes, Group B: 35 eyes). Seventeen eyes in Group A and 13 eyes in Group B had RRD with superior retinal breaks, while 14 eyes in Group A and 19 eyes in Group B had RRD with inferior retinal breaks. There was no statistically difference in preoperative demographic date between the groups. The initial and final anatomical success rates were $97.1 \%$ and $100 \%$ in Group A and $94.3 \%$ and $100 \%$ in Group B, respectively. The success rates between the groups were not statistically different $(p=1)$. The best corrected visual acuity (BCVA) (logMAR) at 12 months after surgery was $-0.02 \pm 0.14$ in Group A and $-0.03 \pm 0.27$ in Group B. The BCVA between the groups was not statistically different $(p=0.27)$.

Conclusions The surgical results of air tamponade were not inferior to 20\% SF6 tamponade in 27GPPV for RRD irrespective of retinal break locations in the present cohort.

\section{Introduction}

Rhegmatogenous retinal detachment (RRD) is a severe vision-threatening condition. Current treatment modalities for RRD include pars plana vitrectomy (PPV) and scleral buckling. Notably, PPV with a wide-angle viewing system has been gaining popularity as the first-line therapy, primarily because of its low invasiveness. Self-sealing small incisions lead to less surgical trauma, postoperative inflammation, and postoperative astigmatism, resulting in better postoperative comfort for patients and an earlier visual recovery [1-13]. However, disadvantages of PPV for RRD include postoperative visual disturbance and limitations in day-to-day activities for an extended period, due to gas tamponade within the vitreous cavity [14]. Typically, sulfur hexafluoride (SF6), octafluoropropane (C3F8), and room air are used as postoperative tamponade materials to seal causal retinal breaks; 20\% SF6 lasts for 2 weeks [14-16], while 13\% C3F8 lasts for $\sim 2$ months $[14,15]$ in the vitreous cavity. In comparison to these materials, room air has a shorter absorption time. Therefore, room air may be useful for reducing the disadvantages of PPV listed above. In addition, tamponade with room air has a lower probability of increased intraocular pressure and a reduced possibility of concurrent cataracts compared to long-acting gases (LAGs), such as SF6 and C3F8 [14].

There have been some reports of exploratory research involving tamponade materials at PPV for RRD [17-23]. Although several studies have reported that PPV with air tamponade provides a primary anatomic success rate comparable to that achieved with LAG tamponade, regardless of retinal break locations, LAG is generally thought to be superior to room air as a tamponade material for postoperative anatomic success after PPV, particularly for patients with RRD with inferior retinal breaks [21, 23]. Thus far, the use of room air remains rather controversial.

In this study, we compared the initial anatomical success rates between room air and 20\% SF6 tamponade after 27gauge (27G) PPV for RRD.

\section{Materials and methods}

This is retrospective analyses of the medical records of 70 eyes of 70 consecutive patients who underwent 27GPPV with air or $20 \%$ SF6 tamponade for primary uncomplicated RRD. All work was approved by the institutional review board in Kobe 
University School of Medicine. The procedure used conformed to the Tenets of the Declaration of Helsinki. Patients were enrolled from July 2016 to December 2017. Patients who had surgery before March 2017 used LAG as a tamponade material, and those who had surgery after April 2017 used air as a tamponade material. Eyes with giant retinal tears, proliferative vitreoretinopathy (PVR), atopic dermatitis or a history of prior surgery for RRD were excluded. All patients were followed-up 12 months after the surgery. The enrolled patients were divided into two groups. Group A comprised patients who underwent the 20\% SF6 tamponade. Group B comprised patients who underwent the air tamponade. The total number of patients was chosen by setting the power $=0.8, \mathrm{a}=0.05$ and the inferiority limit $=0.1$.

The following variables were analysed: sex, age, the number of retinal breaks, locations of retinal breaks, the number of quadrants involved, the presence or absence of the macular detachment, the lens status, the time course changes of best corrected visual acuity (BCVA) expressed as $\log$ MAR, the time course changes of IOP, the postoperative positioning, the primary anatomical success rate, the final anatomical success rate, and the rate of complications. BCVA was measured before and, 1, 3, 6 and 12 months after surgery. The IOP was measured before and, 1 day, 1 week, 1, 3, 6 and 12 months after surgery.

\section{Surgical procedures}

Sub-Tenon anaesthesia was carried out using $\sim 4 \mathrm{ml}$ of mixed $2 \%$ lidocaine and $0.5 \%$ levobupivacaine. The $27 \mathrm{GPPV}$ with a wide-angle non-contact viewing system (Resight ${ }^{\circ}$; Carl Zeiss Meditec AG, Jena, Germany) was performed using the Constellation Vision System (Alcon Laboratories, Inc., Fort Worth, TX, USA). Three cannulas were created with conjunctival displacement and oblique-angled sclerotomies in the inferotemporal, superotemporal, and superonasal quadrants $3.0-4.0 \mathrm{~mm}$ posterior to the limbus. The $27 \mathrm{G}$ chandelier illumination fiber (Oshima vivid, Synergetics, USA, Inc.) was placed at $3.0-4.0 \mathrm{~mm}$ posterior to the limbus for wide-angle intraocular illumination. Before vitrectomy, phacoemulsification and intraocular lens implantation (PEA + IOL) with $2.4 \mathrm{~mm}$ bent transconjunctival single-plane sclero-corneal or clear corneal incision were performed using the same machine for all phakic eyes. Following the core vitrectomy, the vitreous gel was visualized by the injection of triamcinolone acetonide (MaQaid, Wakamoto Pharmaceutical, Tokyo, Japan) during midperipheral vitrectomy. Then, the peripheral vitreous gel was shaved for $360^{\circ}$. Scleral indentation under a wide-angle non-contact viewing system was performed if necessary. No internal drainage retinotomies were made. All retinal detachments were restored intraoperatively. Retinal photocoagulation was applied to completely surround all retinal breaks (Purepoint endoocular probe, Alcon Laboratories, Inc., Fort Worth,
TX, USA) (180 mW, $200 \mathrm{~ms})$. At the end of the surgery, eyes of Group A were flushed with $50 \mathrm{ml}$ of non-expansile gas (20\% SF6) to assure a complete exchange. Additional gas mixture was injected through the pars plana to adjust the IOP, if necessary. Any sclerotomy sites that were found to leak at the end of the surgery were closed with 8-0 vicryl suture. IOP was checked by tactile examination. Subconjunctival corticosteroids were injected, and antibiotic ointment was administered at the end of the surgical procedure. Neither scleral buckle nor perfluorocarbon liquid was used. All patients were encouraged to start the prone positioning as soon as they moved from the operation bed to the wheelchair on the day of the surgery. They were instructed to keep the prone position at the surgery day, followed by the prone position or the supine position for a minimum of 7 days thereafter. The choice of postoperative position was according to a discretion of the surgeon (H.I.).

\section{Statistical methods}

The chi-square test and Fisher's exact probability test for dichotomous variables and an unpaired $t$-test for continuous variables were used to compare the parameters listed above between the two groups. We used the mixed effect model to examine differences in the BCVA and the IOP in each group. For all variables, units reported are the mean value and the standard deviation (SD). Statistical analyses were performed using statistical software (SPSS, version 24.0; IBM Corporation, Armonk, NY). Statistical significance was considered at $p<0.05$.

\section{Results}

Seventy eyes from 70 patients were included in the study, 35 eyes from Group A and 35 eyes from Group B. The perioperative demographic date of the patients from both groups was comparable (Table 1). There were 16 men in Group A and 20 men in Group B $(p=0.34)$. The mean \pm SD age was $56.8 \pm 10.1$ years in Group A and 59.2 \pm 7.9 years in Group B $(p=0.78)$. The mean \pm SD number of retinal breaks was $1.9 \pm 1.6$ in group $\mathrm{A}$ and $2.5 \pm 1.8$ in group B $(p=0.38)$. Seventeen eyes had original breaks in the superior quadrant, 14 in the inferior quadrant and 4 at the 3 or 9 o'clock position in Group A. In contrast, 13 eyes had original breaks in the superior quadrant, 19 in the inferior quadrant and 3 at the 3 or 9 o'clock position in Group B. There was no statistical difference in the retinal break locations between the groups $(p=0.68)$. Thirty-four eyes had RRD involving one or two quadrants, and 1 eye had a more extensive RRD involving three or four quadrants in Group A. Thirty-two eyes had RRD involving one or two quadrants, and 3 eyes had a more extensive RRD 
Table 1 Perioperative demographic date for the patients.

\begin{tabular}{llll}
\hline Characteristics & Group A (SF6) & Group B (Air) & $p$ value \\
\hline No. of eyes & 35 & 35 & - \\
Sex, male/female & $16 / 19$ & $20 / 15$ & 0.34 \\
Age(years), mean \pm SD & $56.8 \pm 10.1$ & $59.2 \pm 7.9$ & 0.78 \\
No. of breaks, mean \pm SD & $1.9 \pm 1.6$ & $2.5 \pm 1.8$ & 0.38 \\
Location of breaks, superior/inferior/3 or 9 o'clock & $17 / 14 / 4$ & $13 / 19 / 3$ & 0.68 \\
Quadrant of retinal detachment, 1/2/3/4 & $13 / 21 / 1 / 0$ & $10 / 22 / 2 / 1$ & 0.98 \\
Macular detachment, macular on/macular off & $16 / 19$ & $19 / 16$ & 0.47 \\
Lens status, phakic/pseudophakic & $27 / 8$ & $30 / 5$ & 0.54 \\
Preoperative BCVA (logMAR), mean \pm SD & $0.47 \pm 0.70$ & $0.37 \pm 0.60$ & 0.56 \\
Preoperative IOP (mmHg), mean \pm SD & $15.1 \pm 3.3$ & $14.1 \pm 4.0$ & 0.27 \\
Postoperative position (prone/spine) & $20 / 15$ & $23 / 12$ & 0.46 \\
Initial anatomical success & $34(97.1)$ & $33(94.3)$ & 1 \\
Final anatomical success & $35(100)$ & $35(100)$ & 1 \\
\hline
\end{tabular}

Numbers in parentheses are percentages

$B C V A$ best corrected visual acuity, $I O P$ intraocular pressure, $S D$ standard deviation

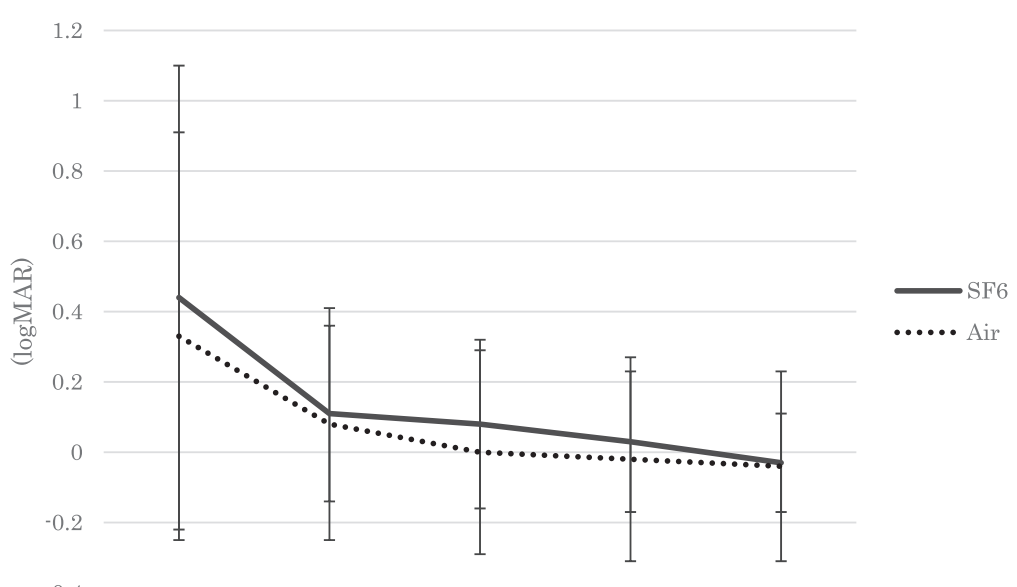

\begin{tabular}{|l|l|l|l|l|l|}
\hline & Pre & 1 month & 3 months & 6 months & 12 months \\
\hline SF6 & $0.47 \pm 0.70$ & $0.10 \pm 0.24$ & $0.08 \pm 0.24$ & $0.03 \pm 0.19$ & $-0.02 \pm 0.14$ \\
\hline Air & $0.37 \pm 0.60$ & $0.09 \pm 0.33$ & $0.01 \pm 0.30$ & $-0.01 \pm 0.30$ & $-0.03 \pm 0.27$ \\
\hline
\end{tabular}

Fig. 1 The change in best corrected visual acuity (BCVA) over time. The mean BCVA $(\log$ MAR) before surgery was $0.47 \pm 0.70$ in Group A and $0.37 \pm 0.60$ in Group B. At 1, 3, 6 and 12 months after surgery, the BCVA was $0.10 \pm 0.24,0.08 \pm 0.24,0.03 \pm 0.19$, and $-0.02 \pm$ 0.14 , respectively, in Group A, and $0.09 \pm 0.33,0.01 \pm 0.30,-0.01 \pm$

involving three or four quadrants in Group B. There was no statistical difference in the extent of RRD between the groups $(p=0.98)$. The macula was attached preoperatively in 16 eyes (45.7\%) in Group A and 19 (54.3\%) in Group B $(p=0.47)$. The number of eyes who received PEA + IOL was 27 eyes in Group A and 30 eyes in Group B $(p=0.54)$. The preoperative log MAR BCVA was $0.47 \pm 0.70$ in Group $\mathrm{A}$ and $0.37 \pm 0.60$ in Group $\mathrm{B}(p=0.56)$. The preoperative IOP $(\mathrm{mmHg})$ was $15.1 \pm 3.3$ in Group $A$ and $14.1 \pm 4.0$ in Group $B(p=0.27)$. The prone positioning was chosen 20 eyes in Group A and 23 eyes in Group B
0.30 and $-0.03 \pm 0.27$, respectively, in Group B. In both groups, the BCVA at $1,3,6$, and 12 months after surgery was statistically better than the preoperative BCVA ( $p<0.01$ for all time points). The BCVA was not statistically different between the groups during the follow-up period $(p=0.56,0.58,0.28,0.26,0.27$, respectively).

( $p=0.46)$. Regarding patients with RRD with inferior retinal breaks, 8 eyes in Group A and 13 eyes in Group B. The spine positioning was chosen 15 eyes in Group A and 12 eyes in Group B. About patients with RRD with inferior retinal breaks, six eyes in Group A and six eyes in Group B. The initial and final anatomical success rates were $97.1 \%$ (34 eyes/ 35 eyes) and $100 \%$ in Group A and $94.3 \%$ (33 eyes/35 eyes) and $100 \%$ in Group $\mathrm{B}$, respectively $(p=1, p=1$, respectively). About patients with RRD with superior retinal breaks, the initial anatomical success rate was $94.1 \%$ (16 eyes/17 eyes) in Group A and 92.3\% (12 eyes/13 eyes) in Group B. 
30

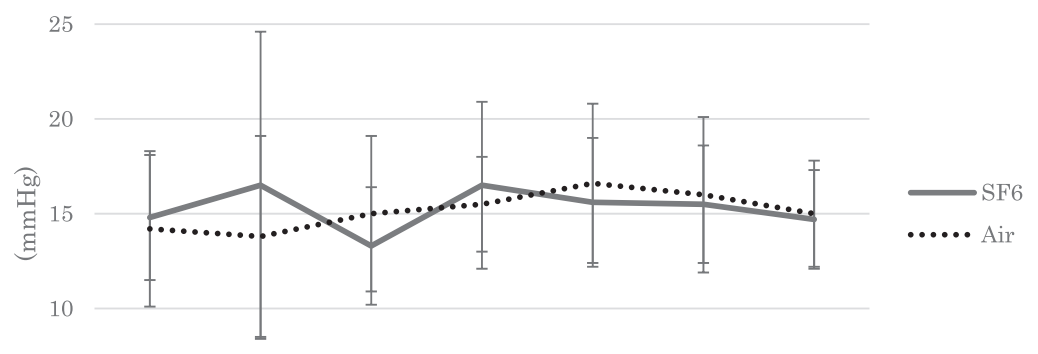

\begin{tabular}{|l|l|l|l|l|l|l|l|}
\hline & Pre & 1 day & 1 week & 1 month & 3 months & 6 months & 12 months \\
\hline SF6 & $15.1 \pm 3.3$ & $16.4 \pm 8.6$ & $13.3 \pm 3.4$ & $16.3 \pm 4.9$ & $15.1 \pm 3.3$ & $15.4 \pm 3.1$ & $14.7 \pm 2.7$ \\
\hline Air & $14.1 \pm 4.0$ & $13.7 \pm 5.4$ & $14.9 \pm 4.0$ & $15.7 \pm 2.6$ & $16.7 \pm 4.3$ & $16.1 \pm 4.3$ & $15.2 \pm 3.2$ \\
\hline
\end{tabular}

Fig. 2 The change in intraocular pressure (IOP) over time. The mean IOP before surgery was $15.1 \pm 3.3 \mathrm{mmHg}$ in Group A and $14.1 \pm 4.0 \mathrm{mmHg}$ in Group B. At 1 day, 1 week, $1,3,6$, and 12 months after surgery, the IOP was $16.4 \pm 8.6,13.3 \pm 3.4,16.3 \pm 4.9,15.1 \pm 3.3$, $15.4 \pm 3.1$ and $14.7 \pm 2.7 \mathrm{mmHg}$, respectively, in Group A, and $13.7 \pm$ $5.4,14.9 \pm 4.0,15.7 \pm 2.6,16.7 \pm 4.3,16.1 \pm 4.3$ and $15.2 \pm 3.2 \mathrm{mmHg}$, respectively, in Group B. In Group A, the IOP at 1 week after surgery was statistically lower than the preoperative IOP $(p=0.02)$ and the
IOP at 1 day, 1, 3, 6, and 12 months after surgery was not statistically different. In Group B, the IOP at 1, 3, and 6 months after surgery was statistically higher than the preoperative IOP $(p=0.04,0.01,0.02$, respectively) and the IOP at 1 day, 1 week, and 12 months after surgery was not statistically different. The IOP was not statistically different between the groups during the follow-up period $(p=0.27$, $0.35,0.67,0.08,0.61,0.17,0.45$, respectively).
Table 2 Details on patients with postoperative complications.

\begin{tabular}{llll}
\hline Complication & Group A (SF6) & $\begin{array}{l}\text { Group } \\
\text { B (Air) }\end{array}$ & Second treatment \\
\hline Retinal redetachment & 1 & 2 & $\begin{array}{l}\text { 27GPPV+gas tamponade } \\
\text { 27GPPV+proliferative membrane } \\
\text { Proliferative vitreoretinopathy }\end{array}$ \\
Macular pucker & 3 & 1 & \\
\hline
\end{tabular}

SF6 sulfur hexafluoride, 27GPPV 27-gauge pars plana vitrectomy, ILM internal limiting membrane
Regarding patients with RRD with inferior retinal breaks, the initial anatomical success rate was $100 \%$ (14 eyes/14 eyes) in Group A and 94.7\% (18 eyes/19 eyes) in Group B.

We next evaluated changes over time in the BCVA and IOP during the follow-up period. Figure 1 showed the change over time in the BCVA. In both groups, the BCVA at $1,3,6$, and 12 months after surgery was statistically better than the preoperative BCVA $(p<0.01$ for all time points). The BCVA was not statistically different between the groups during the follow-up period ( $p=0.56,0.58$, $0.28,0.26,0.27$, respectively). Figure 2 showed the change over time in the IOP. In Group A, the IOP at 1 week after surgery was statistically lower than the preoperative IOP $(p=0.02)$ and the IOP at 1 day, 1, 3, 6, and 12 months after surgery was not statistically different. In Group B, the IOP at 1,3 , and 6 months after surgery was statistically higher than the preoperative IOP $(p=0.04,0.01,0.02$, respectively) and the IOP at 1 day, 1 week, and 12 months after surgery was not statistically different. The IOP was not statistically different between the groups during the followup period $(p=0.27,0.35,0.67,0.08,0.61,0.17,0.45$, respectively).

Table 2 summarizes the details on patients with postoperative complications. Retinal re-detachment occurred in both groups. We performed an additional 27GPPV for the treatment of these complications. All eyes obtained the final retinal attachment. The rate of these complications was similar between the groups.

\section{Discussion}

After PPV for RRD, it is important to ensure that the original retinal breaks remain attached until adhesion is 
achieved between the retina and retinal pigment epithelium following retinal photocoagulation; this prevents the movement of fluid from the vitreous cavity into the subretinal space via the breaks, as well as the shift of residual subretinal fluid toward the breaks [24]. Many previous papers discussed whether air tamponade is sufficient to address these concerns [17-20,22], but the conclusions have remained rather controversial.

Regarding RRD with superior retinal breaks, the filled gas within the vitreous cavity may be able to tamponade the breaks easily because of gravity and buoyancy, regardless of strict postoperative positional restrictions. Moreover, subretinal fluid falls to the inferior quadrant, far from the original retinal breaks, in accordance with gravity. Therefore, it is possible that breaks can be sufficiently sealed for an extended duration postoperatively, even when using air tamponade. In our study, after $27 \mathrm{GPPV}$, the initial anatomic success rate was $92.3 \%$ (12 eyes/13 eyes) in the air tamponade group and $94.1 \%$ (16 eyes/17 eyes) in the 20\% SF6 gas tamponade group, among patients with RRD with superior retinal breaks. These initial anatomic success rates between the two groups were not significantly different ( $p$ $=1$ ). Previous comparative studies showed that the initial anatomic success rates of PPV for RRD were comparable between air tamponade and LAG tamponade groups for patients with RRD with superior retinal breaks [23]. Other reports have also described satisfactory initial anatomic success rates with air tamponade [19, 20, 22]. Hence, we consider air tamponade to be sufficient for the treatment of RRD with superior retinal breaks, in place of LAG tamponade.

However, for patients with RRD with inferior retinal breaks, it may be more difficult to ensure that retinal breaks remain attached for a sufficient duration postoperatively, because it is difficult and burdensome for patients to maintain strict face-down positioning for effective gas tamponade. Moreover, residual subretinal fluid tends to shift toward the inferior part of the subretinal space and may collect around retinal breaks because of gravity. Thus, it is often noted that air tamponade may be unsuitable for treatment of RRD with inferior retinal breaks. In a previous retrospective study, Tan et al. reported that the initial anatomic success rate was significantly different between air tamponade $(81.0 \%)$ and LAG tamponade groups (86.9\%) after 20G- or 25GPPV. (OR 1.97, $\mathrm{p}=0.012$ ) [23]; they suggested that LAG tamponade may be superior to air tamponade for RRD involving inferior quadrants. Conversely, Pak et al. reported that, after 23GPPV or $25 \mathrm{GPPV}$, the initial anatomic success rate for RRD with inferior retinal breaks was $94.4 \%$ in the air tamponade group, which was not significantly different from the $92.9 \%$ observed in the LAG group $(p=1)$ [22]. In their report, perfluorocarbons were used to fully drain subretinal fluid in $30 \%$ of the cases; they asserted that air could be considered for use as a gas tamponade in cases of primary RRD that were managed by sufficient removal of subretinal fluid. MartínezCastillo et al. also reported that 20GPPV with air tamponade achieved a high reattachment rate in RRD with inferior retinal breaks, when perfluorocarbons were used for the removal of subretinal fluid for all patients [17, 18]; they suggested that RRD with inferior retinal breaks could be adequately treated with PPV, even when air tamponade was used, if sufficient drainage of subretinal fluid was performed. In studies using rabbits, a retinal tissue subjected to photocoagulation increased an adhesive strength to $128 \%$ of the strength of normal retina, $24 \mathrm{~h}$ after surgery [24]. In addition, it has been reported that, immediately following reattachment of retinal tissue after detachment, the adhesive strength between the retina and retinal pigment epithelium was comparable to that of normal retina at $24 \mathrm{~h}$ after photocoagulation [24]. Based on those prior findings, we infer that even short-term tamponades may sufficiently reduce the risk of recurrent detachment if the subretinal fluid does not reach the original wound. Thus, sufficient subretinal fluid drainage is essential, especially for patients with RRD with inferior retinal breaks. Although an intentional drainage hole can be created to fully drain the subretinal fluid, there is a risk of visual field impairment after the surgery. Hence, it is likely more desirable to use perfluorocarbon tamponade. However, with respect to RRD with inferior breaks, in the current study, there were 14 eyes in the SF6 gas group and 19 eyes in the air tamponade group. After 27GPPV, the initial success rates were $100 \%$ (14 eyes/14 eyes) in the SF6 gas group and $94.7 \%$ (18 eyes/19 eyes) in the air tamponade group; these rates were not significantly different between the groups, although perfluorocarbon was not used in any eyes. We previously reported the efficacy and safety of postoperative supine positioning after 27GPPV for RRD [25]. From an anatomical perspective, the gas can act better on the peripheral retina in the supine position than in the prone position. In particular, in patients with RRD resulting from inferior retinal breaks, inadequate rest in the prone position can cause the original wound to become the lowest point in the eye, such that subretinal fluid collects at the wound due to gravity. Hence, we consider placing patients in a supine position to be advantageous for the purpose of fully sealing the original retinal breaks through gas tamponade. In addition, the supine position is less demanding for the patient, both physically and psychologically; thus, we suspect that this position is easier for the patient to maintain. In the present study, we proactively imposed a requirement to remain in the supine positioning for patients who were unable to rest sufficiently in the prone positioning. In the present study, $42.9 \%$ (6 eyes/14 eyes) in the SF6 gas group and $31.6 \%$ (6 eyes $/ 19$ eyes) in the air group were chosen the supine positioning. In doing so, even with patients who had air tamponade, we were able to create a situation where the subretinal fluids did not leak into the original breaks, giving 
sufficient duration for the breaks to seal. Thus, we believe that air tamponades are viable alternatives to a LAG, which inevitably lead to temporary vision impairment and restrictions on a patient's daily life, while air tamponades get absorbed more quickly, and patients can maintain an undemanding supine positioning compared with the more strenuous prone positioning that was required when using gas tamponades. We believe it is necessary to conduct studies with greater sample sizes in the future.

The current study had some limitations. First, it was a retrospective study, which may have included a patient selection bias. Additionally, the sample size was small. Largescale, randomized, and prospective studies are warranted.

\section{Conclusion}

We investigated and compared the anatomical success rates, visual outcome and the frequency of complications between air and 20\% SF6 tamponade in 27GPPV for RRD.

Our results demonstrated air tamponade might be not inferior to $20 \%$ SF6 tamponade in 27GPPV for RRD, regardless of retinal break locations.

\section{Summary}

\section{What was known before}

- The use of room air as tamponade material at PPV for RRD remains controversial, particularly RRD with inferior retinal breaks.

\section{What this study adds}

- The surgical results of air tamponade were not inferior to $20 \%$ SF6 tamponade in 27GPPV for RRD irrespective of retinal break locations.

\section{Compliance with ethical standards}

Conflict of interest There is no conflict of interest.

Publisher's note Springer Nature remains neutral with regard to jurisdictional claims in published maps and institutional affiliations.

\section{References}

1. Hakin KN, Lavin MJ, Leaver PK. Primary vitrectomy for rhegmatogenous retinal detachment. Graefe's Arch Clin Exp Ophthalmol. 1993;231:344-6.
2. Escoffery RF, Olk RJ, Grand MG, Boniuk I. Vitrectomy without scleral buckling for primary rhegmatogenous retinal detachment. Am J Ophthalmol. 1985;99:275-81.

3. Hwang JC. Regional practice patterns for retinal detachment repair in the United States. Am J Ophthalmol. 2012;153:1125-8.

4. Jackson TL, Donachie PH, Sallam A. United Kingdom National ophthalmology database study of vitreoretinal surgery: report 3, retinal detachment. Ophthalmology. 2014;121:643-8.

5. Soni C, Hainsworth DP, Almony A. Surgical management of rhegmatogenous retinal detachment: a meta-analysis of randomized controlled trials. Ophthalmology. 2013;120:1440-7.

6. Bourla DH, Bor E, Axer-Siegel R, Mimouni K, Weinberger D. Outcomes and complications of rhegmatogenous retinal detachment repair with selective sutureless 25 -gauge pars plana vitrectomy. Am J Ophthalmol. 2010;149:630-4.

7. Park SW, Kwon HJ, Kim HY, Byon IS, Lee JE, Oum BS. Comparison of scleral buckling and vitrectomy using wide angle viewing system for rhegmatogenous retinal detachment in patients older than 35 years. BMC Ophthalmol. 2015;15:1.

8. Heimann H, Bartz-Schmidt KU, Bornfeld N. Scleral buckling versus primary vitrectomy in rhegmatogenous retinal detachment. A prospective randomized multicenter clinical study. Ophthalmology. 2007;114:2142-54.

9. Campo RV, Sipperley JO, Sneed SR. Pars plana vitrectomy without scleral buckle for pseudophakic retinal detachments. Ophthalmology. 1999;106:1811-5.

10. Tanner V, Minihan M, Williamson TH. Management of inferior retinal breaks during pars plana vitrectomy for retinal detachment. Br J Ophthalmol. 2001;85:480-2.

11. Sharma A, Grigoropoulos V, Williamson TH. Management of primary rhegmatogenous retinal detachment with inferior breaks. Br J Ophthalmol. 2004;88:1372-5.

12. Wickham L, Connor M, Aylward GW. Vitrectomy and gas for inferior break retinal detachments: are the results comparable to vitrectomy, gas, and scleral buckle? Br J Ophthalmol. 2004;88: 1376-9.

13. Chan CK, Lin SG, Nuthi AS, Salib DM. Pneumatic retinopexy for the repair of retinal detachments: a comprehensive review (1986-2007). Surv Ophthalmol. 2008;53:443-78.

14. Thompson JT. Kinetics of intraocular gases: disappearance of air, sulfur hexafluoride, and perfluoropropane after pars plana vitrectomy. Arch Ophthalmol. 1989;107:687-91.

15. Wong RF, Thompson JT. Prediction of the kinetics of disappearance of sulfur hexafluoride and perfluoropropane. Ophthalmology. 1988;95:609-13.

16. Fineberg E, Machemer R, Sullivan P, Norton EW, Hamasaki D, Anderson D. Sulfur hexafluoride in owl monkey vitreous cavity. Am J Ophthalmol. 1975;79:67-76.

17. Martínez-Castillo V, Boixadera A, Verdugo A, García-Arumí J. Pars plana vitrectomy alone for the management of inferior breaks in pseudophakic retinal detachment without facedown position. Ophthalmology. 2005;112:1222-6.

18. Martínez-Castillo VJ, García-Arumí J, Boixadera A. Pars plana vitrectomy alone for the management of pseudophakic rhegmatogenous retinal detachment with only inferior breaks. Ophthalmology. 2016;123:1563-9.

19. Zhang Z, Peng M, Wei Y, Jiang X, Zhang S. Pars plana vitrectomy with partial tamponade of filtered air in Rhegmatogenous retinal detachment caused by superior retinal breaks. BMC Ophthalmol. 2017;17:64.

20. Chen QY, Tang YX, He YQ, Lin HM, Gao RL, Li MY, et al. Air tamponade in retinal detachment surgery followed by ultrawidefield fundus imaging system. Int J Ophthalmol. 2018;11:1198-203.

21. Zhou C, Qiu Q, Zheng Z. Air versus gas tamponade in rhegmatogenous retinal detachment with inferior breaks after 23-gauge 
pars plana vitrectomy: a prospective, randomized comparative interventional study. Retina. 2015;35:886-91.

22. Pak KY, Lee SJ, Kwon HJ, Park SW, Byon IS, Lee JE. Exclusive use of air as gas tamponade in rhegmatogenous Retinal Detachment. J Ophthalmol. 2017;134:1948.

23. Tan HS, Oberstein SYL, Mura M, Bijl HM. Air versus gas tamponade in retinal detachment surgery. $\mathrm{Br} \mathrm{J}$ Ophthalmol. 2013;97:80-82.
24. Folk JC, Sneed SR, Folberg R, Coonan P, Pulido JS. Early retinal adhesion from laser photocoagulation. Ophthalmology. 1989;96:1523-5.

25. Otsuka K, Imai H, Miki A, Nakamura M. Impact of postoperative positioning on the outcome of pars plana vitrectomy with gas tamponade for primary rhegmatogenous retinal detachment: comparison between supine and prone positioning. Acta Ophthalmol. 2018;96:189-94. 